Archives of Clinical and Medical Case Reports

doi: $10.26502 /$ acmcr. 96550042

Volume 2, Issue 6

Case Report

\title{
Linear Lichen and Anterior Cervical Hypertrichosis: Unusual Association
}

\author{
El Anzi Ouiam ${ }^{1 *}$, Maouni Safae ${ }^{1}$, Meziane Mariam $^{1}$, Hassam Badredine $^{1}$ \\ Department of Dermatology-Venereology, Ibn Sina University Hospital, Mohammed V University, Rabat, Morocco
}

"Corresponding Author: Dr. El Anzi Ouiam, Department of Dermatology-Venereology, Ibn Sina University Hospital, Mohammed V University, Rabat, Morocco, Tel: 00212624604105; E-mail: elanzi.ouiam@ gmail.com

Received: 12 October 2018; Accepted: 24 October 2018; Published: 07 November 2018

\begin{abstract}
Linear lichen planus is a rare variety of lichen planus characterized by lesions following the lines of Blaschko. Anterior cervical hypertrichosis is a rare and unrecognized form of local congenital hypertrichosis. It is characterized by the presence of a tuft of terminal hair on the anterior cervical region. We report an exceptional association of these two entities.
\end{abstract}

Keywords: Linear lichen planus; Anterior cervical hypertrichosis; Rare; Association

\section{Case Report}

A 25-year-old woman presented to the Department of Dermatology with a 1-year history of a slightly itchy, hyperpigmented papular, and atrophic linear skin lesions on the back of the right hand and right forearm up to the elbow, and linear hyperpigmented macules under mandibular straight. The physical examination also showed tuft of terminal hair, a few centimeters long, on the anterior and medial aspect of the neck facing the cricoid cartilage. There was no hypertrichosis in the other body regions. Scalp, nails, and mucous membranes were not affected. Histopathologic examination of the hyperpigmented lesion was in favor of lichen. Topical corticosteroids and emollients were applied to the skin lesions (Figure 1 and 2). 


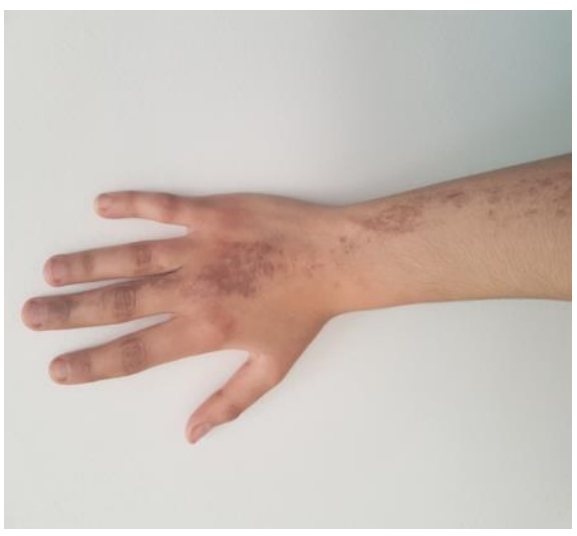

Figure 1: Linear lichenoid lesions on the right arm.

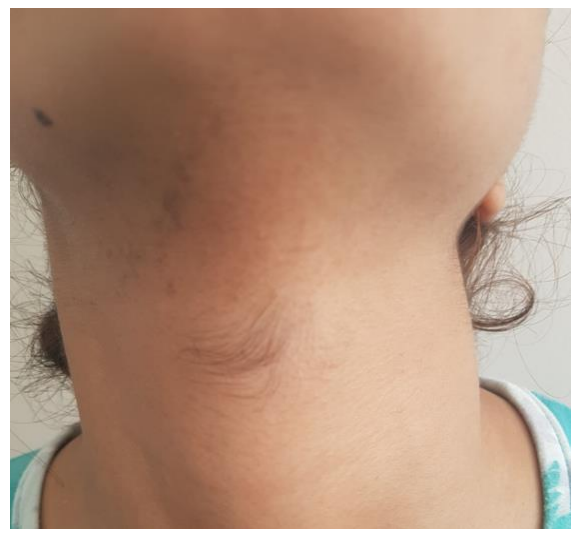

Figure 2: Multiple terminal hair on the laryngeal prominence.

\section{Comments}

Linear lichen planus (LLP) is a form of lichen planus characterized by a linear distribution or zosteriform lesions. It is rare, interesting 0.2 to $0.62 \%$ of cases [1]. Without treatment, the evolution of the LLP is chronic and persistent. Under local or general corticosteroids (at a dose of $0.5 \mathrm{mg} / \mathrm{kg}$ per day of prednisone), the lesions regress generally without relapse [2]. Anterior cervical hypertrichosis (HCA) is a rare dermatologic condition. To date, 42 cases have been reported. Localized hypertrichosis can be congenital or acquired. Acquired lesions usually occur in response to skin inflammation or trauma. Congenital lesions can occur as a primary lesion without any extracutaneous manifestations or as a secondary lesion in the setting of a smooth muscle hamartoma or other congenital malformation [3].

Primary congenital localized forms of hypertrichosis usually arise on otherwise normal skin and often affect specific sites including the elbows, palms and soles, eyebrows, anterior neck, posterior neck, and lower lumbar spine. HCA is considered as a benign pathology. In almost 33\% of the observations, the possibility of associated extra-skin lesions has been described as central or peripheral neurological abnormalities. The possibility of other associated affections is more rare such orthopedic abnormalities, ocular and autoimmune diseases. Association with linear 
plane lichen has never been described [4]. The treatment of HCA is symptomatic, based on the camouflage (hair discoloration), shaving or hair removal definitive or not (chemical depilatory creams, electrolysis, pulsed light or depilatory lasers). It depends on the degree of hypertrichosis and psychosocial context.

\section{Conclusion}

The association of anterior cervical hypertrichosis and LLP might be coincidental. So far, no case of LLP associated with anterior cervical hypertrichosis has been reported in the literature.

\section{References}

1. Kabbash C, Laude TA, Weinberg JM, et al. Lichen planus in the lines of Blaschko. Pediatr Dermatol 19 (2002): 541-545.

2. Krasowska D, Pietrzak A, Lecewicz-Torun B. Unilateral multiple linear lichen planus following the Blaschko lines recurring after deliveries. Dermatology 202 (2001): 340.

3. Nanda A, Al-Aradi I, Ali MT, et al. Anterior cervical hypertrichosis (hairy throat): is it a sign to worry about?. Clin Exp Dermatol 32 (2007): 112-114.

4. Corona-Rivera JR, Gonza' lez-Abarca S, Herna'ndez-Rocha J, et al. Mental retardation in a boy with anterior cervical hypertrichosis. Am J Med Genet 135 (2005): 69-71.

Citation: El Anzi Ouiam, Maouni Safae, Meziane Mariam, Hassam Badredine. Linear Lichen and Anterior Cervical Hypertrichosis: Unusual Association. Archives of Clinical and Medical Case Reports 2 (2018): 187-189.

(C) 1 BY $\begin{aligned} & \text { This article is an open access article distributed under the terms and conditions of the } \\ & \text { Creative Commons Attribution (CC-BY) license 4.0 }\end{aligned}$ 